\title{
Ueber Darstellung von Lecithin und anderen Myelinsubstanzen aus Gehirn- und Eigelbextracten.
}

Von

\author{
Dr. G. Zuelzer, \\ derz. Assistenten der med. Klinik Giessen.
}

(Aus dem Laboratorium des Herrn Dr. Blum-Frankfurt a. M.)

(Der Redaction zugegangen am 4. April 1899.)

Es ist mir gelungen, im Aetherextract des Gehirns neue Myelinsubstanzen neben den bisher schon bekannten aufzufinden und mittelst eines einfachen Verfahrens, bei dem Zersetzungen ausgeschlossen scheinen, $\mathrm{zu}$ isoliren. Die ausführliche Beschreibung der Methode zur Darstellung des neuen Gehirnbestandtheils, sowie die Angaben einiger Eigenschaften des neuen Körpers, finden sich weiter unten gegeben; hier möchte ich nur ganz kurz das Wesentliche des Untersuchungsganges skizziren. Bisher haben alle Forscher bei der Bearbeitung des Aetherextractes des Gehirns dadurch unter ungünstigen und complicirten Versuchsbedingungen zu leiden gehabt, dass ihnen die Abscheidung des Cholesterins entweder nur unvollkommen oder doch nur mit bedeutenden Substanzverlusten durch vielfaches Umlösen gelang. Ich habe nun in dem Aceton ein einfaches Mittel gefunden, um von vornherein die Bestandtheile des Aetherextractes fast quantitativ in 2 Gruppen zu zerlegen: die cholesterinhaltige Aetheracetonlösung und den cholesterinfreien Niederschlag.

Versetzt man einen nicht allzu verdünnten Aetherextract von Gehirn mit Aceton, so fällt ein voluminöser weiss-gelber Niederschlag aus. Derselbe erweist sich als frei von Cholesterin; welches in der Aetheracetonlösung verbleibt, enthält aber 
mehrere P-haltige Antheile. Die letzteren sind leicht dadurch von einander zu trennen, dass der eine von ihnen, das Protagon, in cholesterinfreiem Aether nicht mehr löslich ist. Der zweite, in reinem Aether lösliche Antheil lässt sich durch Versetzen mit Alkohol im Ueberschuss nochmals in zwei Theile scheiden: den in Lösung bleibenden, das Lecithin, und einen zweiten, der durch Alkohol gefällt wird. Letzterer Niederschlag, der Nund P-haltig ist, enthält den neu aufgefundenen Körper.

Da mir anfänglich die Bearbeitung des Gehirnextractes mit Aceton beträchtliche Schwierigkeiten bot, so studirte ich zunächst die Acetonfällung im Aetherextract des Eigelbs, in der Vermuthung, dort weniger complicirte Verhältnisse zu finden. Wenngleich hierbei sich nichts wesentlich Neues gezeigt hat, so bietet diese Behandlung des Eigelbs doch eine bequeme Methode zur Darstellung des Lecithins, so dass eine kurze Mittheilung derselben am Schlusse gerechtfertigt erscheint: Die Aufschliessung des Acetonniederschlages ergab für das Eigelb, dass derselbe sich aus Lecithin und Palmitin zusammensetzt. Beide Bestandtheile konnten durch Alkohol getrennt werden.

Im Einzelnen gestaltet sich die Gehirnuntersuchung folgendermassen: Das Hirn eines frisch geschlachteten Ochsen wird unmittelbar nach seiner Herausnahme, nachdem die Hirnhaut möglichst vollständig abgezogen ist, zerschnitten und in Aether suspendirt. Durch geeignete Unterlagen (Watte oder dergleichen) ist dafür zu sorgen, dass die Gehirnmasse nicht unmittelbar dem Boden des Gefässes aufliegt. Es bildet sich hierbei, wie schon Baumstark ${ }^{1}$ ) es beschreibt, eine untenliegende Blutschicht und ein darüber stehender, gelb gefärbter Aetherextract; beide werden abgegossen und im Scheidetrichter getrennt, und dieses Verfahren mit neuem Aether vielfach wiederholt. Die Aetherextraction wird so lange fortgesetzt, bis der zur Probe entnommene Aether beim Verdunsten keinen Rückstand mehr hinterlässt.

Auf die weitere Behandlung des äthererschöpften Gehirns mit $80 \%$ igem Alkohol bei $45^{\circ}$ C. soll hier nicht näher ein-

1) Zeitschr. f. physiol. Chemem., Bd. IX., S. 162 ff. 
gegangen werden. Sie ist verschiedentlich ausführlich beschrieben worden, ${ }^{1}$ ) und sie liefert bekanntlich nur eine einzige Substanz, das Protagon. Die Eigenschaften des letzteren finden sich in der erwähnten Ruppel'schen Arbeit eingehend geschildert.

Die vereinigten, ziemlich klaren Aetherfiltrate werden entweder im Evacuationsapparat eingeengt oder in einer grossen Schale der Verdunstung an der Luft bei gewöhnlicher Temperatur überlassen. Es fällt hierbei ein fast schneeweisser, ziemlich massiger, feinflockiger Niederschlag aus. Derselbe ist P-haltig und erweist sich, wie schon Frémy und Baumstark ${ }^{2}$ ) beobachteten, als identisch mit dem aus dem Alkoholextract darstellbaren Protagon. Eine Analyse dieses aus Alkohol von $45^{\circ}$ umkrystallisirten Protagons 'ergab folgendes Resultat:

$$
\begin{gathered}
\mathrm{C}=66,90 \% \\
\mathrm{H}=11,59 \% \\
\mathrm{~N}=3,30 \% \\
\mathrm{P}=1,01 \% \\
\mathrm{~S}=
\end{gathered}
$$

Kohlenstoff-Wasserstoff-Bestimmung mit Bleichromat unter Vorlegung reducirter Kupferspiralen:

0,1710 gr. Substanz gaben 0,4195 gr. $\mathrm{CO}_{z}=66,9 \% \mathrm{C}$ und $0,178 \tilde{\text { gr. }}$

$$
\mathrm{H}_{8} \mathrm{O}=11,59 \% \mathrm{H} \text {. }
$$

Stickstoffbestimmung nach Dumas:

0,1810 gr. Substanz gaben bei $16^{\circ}$ C. und $749 \mathrm{~mm}$. Barometerstand $\tilde{5}, 2 \mathrm{ccm} .=0,00597$ gr. $=3,30 \% \mathrm{~N}$.

Phosphorbestimmungen. (Sämmtliche Aschebestimmungen wurden nach der Methode von Carius im zugeschmolzenen Rohr ausgeführt.)

$$
0,3442 \text { gr. Substanz gaben } 0,0124 \text { gr. } \mathrm{Mg}_{2} \mathrm{P}_{2} \mathrm{O}_{7} \text {. }
$$

Der Stickstoffgehalt dieses aus dem Aetherextract dargestellten Protagons $(3,30 \%)$ stimmt sehr gut mit dem N-Gehalt, den ich durch Analysen bei dem aus dem Alkoholextract gewonnenen Protagon ermittelte: $N=3,27 \%$ u. $=3,05 \%$.

1) 1. c. und Ruppel, Zeitschr. f. Biolog., N. F., 13, S. 96, woselbst die übrige Litteratur angegeben ist.

2) 1 . c. 
I. 0,3190 gr. Substanz gaben bei $15^{\circ}$ C. und $756 \mathrm{~mm}$. Barometerstand $8,9 \mathrm{ccm} .=0,01036$ gr. $=3,27 \% \mathrm{~N}$.

II. 0,2780 gr. Substanz gaben bei $18^{\circ} \mathrm{C}$. und $758 \mathrm{~mm}$. Barometerstand $7,4 \mathrm{ccm} .=0,0084 \mathrm{i} \mathrm{gr} .=3,05 \%$.

In der Litteratur zeigen nur die Kossel'schen Analysen ${ }^{1}$ ) des Protagons einen mit meinen Resultaten übereinstimmenden Stickstoffgehalt (im Mittel 3,25\%), während die anderen Autoren bedeutend niedrigere Werthe gefunden haben (Liebreich $2,80 \%$, Gamgee und Blankenhorn 2,39\%, Baumstark 2,35\%, Ruppel 2,32\%). Schwefel habe ich im Protagon nicht nachweisen können.

Nach dem Einengen des Aetherextractes und dem Abfiltriren von dem ausgefallenen Protagon wird die jetzt klare ätherische Lösung mit Aceton im Ueberschuss versetzt, solange noch ein Niederschlag entsteht. Der Niederschlag, welcher abfiltrirt und sorgfältig mit Aceton gewaschen wird, erweist. sich als völlig frei von Cholesterin, ${ }^{2}$ ) während die gesammte Masse des Cholesterins sich im Aceton-Aetherfiltrat in fast reinem Zustande befindet. Kocht man nämlich den durch Abdestilliren des Acetonäthers gewonnenen Rückstand mit Alkohol und filtrirt die heisse Flüssigkeit, so bleibt auf dem Filter meist nur eine ganz geringfügige Menge eines gelblichen Körpers (wahrscheinlich kleine, der Fällung mit Aceton entgangene Reste der später zu beschreibenden Myelinsubstanzen), während im erkaltenden Alkoholfiltrat reines Cholesterin vom Schmelzpunkt $145^{\circ}$ auskrystallisirt. Auch Lecithin kann bei der Abscheidung mit Aceton nicht in grösserer Menge der Fällung entgangen sein, denn das Alkoholfiltrat liefert mit $\mathrm{PtCl}_{4}$ kaum mehr als eine leichte Trübung.

Wir haben also in der Acetonfällung eine Methode, das Cholesterin auf eine ganz einfache, nicht eingreifende Art von den übrigen Bestandtheilen des Aetherextractes zu trennen.

Zur Weiterbehandlung des Acetonniederschlages, den ich hier den Uebersicht halber als Nd bezeichnen will, wird der-

1) Zeitschr. f. physiol. Chem., Bd. XVII, S. 438.

2) Auflösen in $\mathrm{CHCl}_{3}$, Ringprobe mit $\mathrm{H}_{2} \mathrm{SO}_{4}$. 
selbe, nach sorgfältigem Waschen mit Aceton, mit Aether aufgenommen, wobei ein Theil desselben ungelöst bleibt $\left(\mathrm{Nd}_{\mathbf{u}}\right)$, der in Lösung gehende Antheil sei als $\mathrm{Nd}_{1}$ bezeichnet. Nach den Angaben früherer Autoren war es wahrscheinlich, dass der jetzt in Aether unlösliche Körper Protagon ist, welches vorher in dem stark eingeengten Aetherextract noch durch Cholesterin in Lösung gehalten worden war. Er zeigte in der That alle Eigenschaften jenes Körpers und liess sich in Alkohol von $45^{\circ}$ umkrystallisiren, um beim Erkalten in den bekannten rosettenförmig angeordneten Nadeln auszufallen. Da ausserdem die Analyse dieser aus Alkohol von $45^{\circ}$ umkrystallisirten Substanz $\left(\mathrm{Nd}_{\mathrm{u}}\right)$ einmal nach Dumas einen $\mathrm{N}$-Gehalt von $3,25 \%$ ergab, ein anderes Mal aber nach Kjeldahl aus 0,972 gr. Substanz $2,8 \check{\%} \% \mathrm{~N}$, scheint mir ihre Natur als Protagon sichergestellt.

\section{Analyse nach Dumas:}

0,1821 gr. Substanz gaben bei $29^{\circ} \mathrm{C}$. und $753 \mathrm{~mm}$ Barometerstand

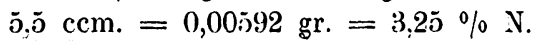

Falls überhaupt Lecithin selbständig im Gehirn vorkommt - und die Ansichten darüber sind in der Litteratur anscheinend sehr getheilt - -, so müsste es sich in dem ätherlöslichen Theil $\left(\mathrm{Nd}_{1}\right)$ des Acetonniederschlages vorfinden, da, wie unsere Vorstudien am Aetherextract des Eigelbes ergeben hatten, Lecithin sich selbst aus fett- und cholesterinhaltigen Aetherlösungen leicht durch Aceton niederschlagen lässt. Der Nachweis reinen Lecithins hinwiederum stellte sein selbständiges isolirtes Vorkommen im Gehirn sicher; denn die bis jetzt mit der Gehirnmasse vorgenommenen chemischen Operationen Lösen in Aether, Fällen mit Aceton - sind so indifferenter Natur, dass selbst die einfachste Spaltung dadurch ausgeschlossen erscheint. Zum Nachweis des Lecithins wurde der ätherlösliche Theil des Acetonniederschlages $\left(\mathrm{Nd}_{\mathrm{l}}\right)$ mit Alkohol versetzt. Dabei entsteht eine starke Fällung $\mathrm{Nd}_{\mathrm{lu}}$; die alkoholische Lösung sei mit $\mathrm{Nd}_{l l}$ bezeichnet. Letztere, in welcher das Lecithin $\mathrm{zu}$ suchen war, gab nun in der That mit Platinchlorid einen, aber nicht sehr reichlichen, Niederschlag. Derselbe 
enthielt nach vollkommener Trocknung 10,7\% Pt. Lecithin (als Dipalmityllecithin angenommen) verlangt einen $\mathrm{Pt}-G e h a l t$ von $10,87 \%$.

Die alkoholische Lösung gab ferner nach genügender Einengung einen Niederschlag mit Aceton (cf. unten Lecithindarstellung aus Eigelb) und nach dem Abdestilliren des Alkohols blieb eine zähe, wachsartige, knetbare Substanz von der Beschaffenheit des Lecithins zurück. Dieselbe war P-haltig; eine quantitative P-Analyse wurde nicht ausgeführt. Es dürfte somit das freie Vorkommen von Lecithin im Gehirn gesichert sein.

Der nunmehr $\mathrm{zu}$ besprechende recht reichliche Alkoholniederschlag des ätherlöslichen Theils des ersten Acetonniederschlages $\left(\mathrm{Nd}_{\mathrm{lu}}\right)$ - einem Ochsenhirn entsprechen meiner Schätzung nach etwa 3 gr. der trockenen Substanz - imponirte anfangs als ein einheitlicher Körper. Er schien, frisch gefällt, sich vollkommen wieder in Aether lösen zu können, um durch. Alkohol oder Aceton von Neuem niedergeschlagen zu werden, und gab sehr gut übereinstimmende $\mathrm{C}$-, $\mathrm{H}$ - und P-Analysenwerthe. Nur die in weiteren Grenzen $(4-6 \% / 0)$ schwankenden $\mathrm{N}$-Werthe liessen an die Möglichkeit denken, dass 2 Körper von zwar ähnlichem $\mathrm{C}$-, $\mathrm{H}$ - und $\mathrm{P}$-, aber verschiedenem $\mathrm{N}$-Gehalt in dem Niederschlag enthalten seien. Genauere Beobachtung lehrte dementsprechend alsbald, dass ein Theil schneller als der andere in Lösung ging, und dass ssich bei öfterem Umlösen allmählich ein geringer Theil abtrennen liess, der für sich nicht mehr in Aether löslich war. Die Abscheidung bietet nicht unerhebliche Schwierigkeiten, da der feine Niederschlag das Filter leicht passirt und deshalb nur durch Decantiren abgesondert werden kann. Fraglos gelingt jedoch eine Reinigung mittelst öfteren Lösens in Aether und Fällens mit Alkohol oder Aceton. Auch hier dürfte, wie beim Protagon, eine Veränderung der Aetherlöslichkeit der einen Substanz durch die Gegenwart der anderen hervorgerufen sein. Lässt man die Alkohol- oder Acetonfällung längere Zeit mit Alkohol oder Aceton stehen oder filtrirt man den Niederschlag $a b$ und lässt ihn lufttrocken werden, so ändert sich die Löslichkeit des Anfangsniederschlages in noch höherem Maasse, als eben beschrieben, der grössere Theil bleibt zwar nach wie vor löslich 
und liefert den früheren gleichwertige Analysen; ein kleinerer Theil aber ist nicht mehr in Aether zu lösen, zumeist aber noch in Chloroform oder Benzol. Auf diese Weise aus einer Lösung mit Alkohol oder Aceton niedergeschlagen und zur Analyse gebracht, ergab der zweite Körper einen C-Gehalt von $55 \%$ und noch niedriger, also um ca. $5 \%$ geringer, als der ätherlösliche Antheil, hingegen einen N-Gehalt bis nahezu $11 \%$.

Diese Substanz (b), die nach vollkommenem Trocknen auch in Benzol und Chloroform nicht mehr löslich ist, stellte die Verunreinigung des ätherlöslichen Theils a dar. Hier müssen neue Untersuchungen einsetzen, um, sei es durch fractionirte Fällung oder durch ein neues und andersartiges Fällungsmittel, eine sichere und vollkommene Trennung der Substanzen a und $b$ herbeizuführen.

Der Körper a aber, der Hauptantheil des Alkoholniederschlages $\mathrm{Nd}_{l u}$, kann, wie ich annehmen möchte, selbst in reinster Form kaum nennenswerth andere Analysenwerthe ergeben, als die von mir gefundenen, und zwar sind die höchsten $\mathrm{C}$ - und die niedrigsten $\mathrm{N}-\mathrm{W}$ erthe als die den richtigen am nächsten liegenden anzusehen. Es folgen die Analysen tabellarisch geordnet:

\begin{tabular}{c|c|c|c|c}
\hline Körper & $\mathrm{C}$ & $\mathrm{H}$ & $\mathrm{N}$ & $\mathrm{P}$ \\
\hline I & 60,27 & 9,87 & $\begin{array}{l}5,5 \\
5,6\end{array}$ \\
& & $\begin{array}{l}5,6 \\
5,7 \mathrm{Kjeldahl}\end{array}$ & 2,98 \\
\hline II & 60,26 & 9,82 & $\begin{array}{l}4,04 \mathrm{Dumas} \\
4,04 \text { D } \\
3,80 \mathrm{Kjeldahl}\end{array}$ & 2,61 \\
\hline III & 60,04 & 9,53 & $\begin{array}{l}4,2 \mathrm{Dumas} \\
3,9\end{array}$ & 2,85 \\
\hline IV & 59,37 & 9,36 & $6,2 \mathrm{Kjeldahl}$ & 2,52 \\
\hline
\end{tabular}

Dreimal gelang es, den als b bezeichneten, also nach dem Trocknen in Aether nicht mehr löslichen Körper in genügender Menge zur Analyse zu gewinnen; leider reichte sie nicht mehr zur P-Bestimmung. 
$\mathrm{V}$ ist von Körper IV durch Trocknen an der Luft abgetrennt, doch enthält IV, wie der hohe $\mathrm{N}$ - und der ensprechend niedrige C-Gehalt beweist, noch beträchtliche Mengen von b.

$\begin{array}{lcccl} & \mathrm{C} & \mathrm{H} & \mathrm{N} \\ \mathrm{V} & \overline{5} 5,52 & 8,74 & 10,97 \text { (Kjeldahl) } \\ \text { VI } & - & - & 10,6 \cdot \text { (Dumas) } \\ \text { VII } & 55,83 & 9,03 & -\end{array}$

Nach diesen Analysenergebnissen erscheint mir die Annahme die grösste Berechtigung zu besitzen, dass dem in Aether leicht löslichen Antheil die Zusammensetzung

zukommt.

$$
\begin{aligned}
& \mathrm{C}=60,2 \% \\
& \mathrm{H}=9,8 \% \\
& \mathrm{~N}=3,8 \% \\
& \mathrm{P}=2,6 \% \\
& \mathrm{O}=23,6 \%(\mathrm{~S}=0)
\end{aligned}
$$

Es ist ja natürlich nicht ganz von der Hand zu weisen, dass der $\mathrm{N}$-Gehalt noch etwas niedriger, der $\mathrm{C}$ - und $\mathrm{H}$-Gehalt aber höher sein könnte: es spricht aber dagegen der Umstand, dass auch öfteres Umlösen der Substanz a keine geringeren Werthe ergab als ca. $4 \% \mathrm{~N}$ und $60 \% \mathrm{C}$; wie z. B. beim Präparat II, das 4 mal in Aether gelöst und abwechselnd mit Alkohol und Aceton ausgefällt wurde, während Präparat III nur 2 mal in dieser Weise behandelt wurde; und doch ergaben beide Präparate fast identische Analysenzahlen.

Die Annahme, dass Substanz VI etwa bei dem Trocknungsprocess aus a abgespalten wurde, besitzt keine Wahrscheinlichkeit, da mehrere Präparate noch nach Monaten dieselben Werthe bei der Analyse lieferten, wie bei der ursprünglichen Untersuchung, obwohl sie in der Zwischenzeit lange im VacuumExsiccator verweilt hatten.

Ein Blick auf die Tabelle zeigt ein Schwanken des $\mathrm{N}$-Gehalts der Myelinsubstanz a zwischen 4 und 6\%, während der C- und H-Gehalt gut übereinstimmt und nur einmal um ca. 0,7 resp. $0,4 \%$ niedriger liegt, und zwar enthält dasjenige Präparat am wenigsten $\mathrm{C}$ und $\mathrm{H}$, das sich andererseits als das N-reichste erwiesen hat. Das lässt sich wohl so erklären, dass der Substanz a jener zweite Körper $b$ in wechselnden 
Mengen noch angehaftet hat. Der C- und H-Gehalt dieser Beimengung liegt ja demjenigen der Hauptsubstanz so nahe, dass die Analyse durch geringe Beimischungen dersetben kaum verändert werden kann; der $\mathrm{N}$-Gehalt aber ist immerhin so verschieden, dass hier schon kleinere Mengen von $b$ einen bemerkbaren Ausschlag zu geben vermögen. Nimmt man z. B. an, dass 1 Theil von $b 10$ Theilen a beigemengt ist - in Wirklichkeit dürfte die Verunreinigung wohl noch erheblich geringer sein -, so erhalten wir, wenn

$$
\begin{aligned}
& \mathrm{a}=60,26 \% \mathrm{C}, \quad 9,82 \% \mathrm{H}, \quad 3,8 \% \mathrm{~N}, \quad 2,61 \% \mathrm{P} \\
& \begin{aligned}
\mathrm{b} & =55,52 \% \mathrm{C}, \quad 8,74 \% \mathrm{H}, \quad 10,97 \% \mathrm{~N}, \quad 2,52 \% \mathrm{P} \\
10 \mathrm{a}+\mathrm{b} & =59,83 \% \mathrm{C}, \quad 9,72 \% \mathrm{H}, \quad 4,45 \% \mathrm{~N}, \quad 2,60 \% \mathrm{P}
\end{aligned}
\end{aligned}
$$

selbst bei nur 5 Theilen a auf 1 Theil $\mathrm{b}$ erhielten wir noch

$$
\begin{aligned}
& \mathrm{C}=59,47 \% \\
& \mathrm{~N}=4,995 \%,
\end{aligned}
$$

also Werthe, die nur bezüglich des $\mathrm{N}$-Gehalts einer Correctur bedürfen; und zwar würden wir, wie schon erwähnt, den niedrigsten $\mathrm{N}$-Gehalt als den richtigsten anzusehen haben.

Der oben analysirte Körper a (I - IV) stellt trocken eine weissliche Masse dar, die, vor Luft geschützt, lange Zeit ihre helle Farbe beibehält, dem Lichte ausgesetzt aber bald eine lehmgelbe Färbung annimmt. Ein Schmelzpunkt besteht nicht; bei $128^{\circ}$ beginnt die Zersetzung der Substanz unter allmählicher Gasentwicklung. Der Körper löst sich in Aether, Benzol, Chloroform und wird durch Alkohol oder Aceton niedergeschlagen.

Erst eine Erschliessung der Constitution der nunmehr leicht zugänglich gemachten neuen Gehirnsubstanzen wird in Zukunft die richtige Classification und damit ihren Namen von selbst ergeben.

Anmerkung. Der oben beschriebene Körper gehört nach Thudichum's Eintheilung in die Gruppe seiner Kephaline und die Analyse desselben zeigt auch eine gewisse Aehnlichkeit mit der des Kephalins. Die Art seiner Darstellung ist principiell von der Thudichum's verschieden; doch ist hier nicht der Ort. auf diese bis in die letzte Zeit fortgeführte Streitfrage bezüglich der Thudichum'schen Darstellungsmethode einzugehen. Es sei nur Thudichum's Analyse des Kephalins mitgetheilt (cf. Thudichum, anatomische und klinische Chemie): $\mathrm{C}=60 \%$, $\mathrm{H}=9,39 \%, \mathrm{~N}=1,68 \%, \mathrm{P}=4,27 \%, \mathrm{O}=24,66 \%$. Das Kephalin

Hoppe-Seyler's Zeitschrift f. physiol. Chemie, XXVII. 18 
besitzt demnach, verglichen mit obigem Körper, bei annähernd gleichem $\mathrm{C}-\mathrm{H}$ - und $\mathrm{O}-\mathrm{Gehalt}$ einen bedeutend geringeren $\mathrm{N}$ - und höheren P-Gehalt.

Die von Thudichum angeführten Eigenschaften des Kephalins, dass die ätherische Lösung über Nacht roth werde, beim Eindampfen sich bräune, dass sie grün fluorescire, dass das einige Zeit trocken aufbewahrte Kephalin eine dunkelschwarzgrüne Reflexfarbe annehme, alles dies war bei dem oben beschriebenen Körper nicht zu beobachten.

Nach obigen Ausführungen glaube ich mich zu dem Schlusse berechtigt, dass in dem Acetonniederschlag des Gehirnätherextractes neben dem bisher bekannten Protagon noch Lecithin und zwei neue Myelinsubstanzen enthalten sind.

Analysenbelege.

I $\quad 0,4660 \mathrm{gr}$. Substanz ergaben

$0,4169 * \mathrm{H}_{2} \mathrm{O}$ und $1,0296 \mathrm{gr}$. $\mathrm{CO}_{2}$, woraus

$\mathrm{C}=60,27 \%, \mathrm{H}=9,87 \%$.

II 0,4268 gr. Substanz ergaben

0,3755 \& $\mathrm{H}_{2} \mathrm{O}$ und $0,9430 \mathrm{gr}$. $\mathrm{CO}_{2}$, woraus

$\mathrm{C}=60,26 \%, \mathrm{H}=9,82 \%$.

III 0,2477 gr. Substanz ergaben

0,2125 - $\mathrm{H}_{2} \mathrm{O}$ und $0,5453 \mathrm{gr} . \mathrm{CO}_{2}$, woraus

$\mathrm{C}=60,04 \%, \mathrm{H}=9,53 \% \%$.

IV 0,2545 gr. Substanz ergaben

0,2145 × $\mathrm{H}_{2} \mathrm{O}$ und $0,55 \mathrm{f0} \mathrm{gr}$. $\mathrm{CO}_{2}$, woraus

$\mathrm{C}=59,97 \%, \mathrm{H}=9,36 \%$.

$\mathrm{V} \quad 0,22: 30$ gr. Substanz ergaben

0,1755 » $\mathrm{H}_{2} \mathrm{O}$ und $0,4540 \mathrm{gr} . \mathrm{CO}_{2}$, woraus

$\mathrm{C}=55,52 \%, \mathrm{H}=8,74 \%$.

VII 0,3475 gr. Substanz ergaben

0,3072 » $\mathrm{H}_{2} \mathrm{O}$ und $0,7072 \mathrm{gr} . \mathrm{CO}_{2}$, woraus

$\mathrm{C}=55,83^{\circ} \%, \mathrm{H}=9,03 \%$.

P-Bestimmungen.

I 0,3566 gr. Substanz ergaben 0,0380 gr. $\mathrm{Mg}_{2} \mathrm{P}_{2} \mathrm{O}_{\bar{\tau}}$ $\mathrm{P}=2,98^{\circ} \%$.

II 0,4303 gr. Substanz ergaben 0,0402 gr. $\mathrm{M}_{2} \mathrm{P}_{2} \mathrm{O}_{\tau}$ $\mathrm{P}=2,61 \%$.

III 0,4554 gr. Substanz ergaben 0,0465 gr. $\mathrm{Mg}_{2} \mathrm{P}_{2} \mathrm{O}_{\tau}$ $\mathrm{P}=2,85^{\circ} \%$.

IV 0,456 gr. Substanz ergaben 0,0410 gr. $\mathrm{Mg}_{2} \mathrm{P}_{2} \mathrm{O}_{7}$ $\mathrm{P}=2,52 \%$.

$\mathrm{N}$-Bestimmungen nach Dumas.

Ia $0,4790 \mathrm{gr}$. Substanz gaben bei $769 \mathrm{~mm}$. und $14^{\circ}$ $22,2 \mathrm{ccm} .=0,02644 \mathrm{gr} .=5,50 \% \mathrm{~N}$. 
Ib $0,5895 \mathrm{gr}$. Substanz gaben bei $7 \check{2} \mathrm{~mm}$. und $17,5^{\circ}$

$28,8 \mathrm{ccm} . \mathrm{N}=0,0329 \mathrm{gr} .=5,6 \% \mathrm{~N}$.

Ic $0,4777 \mathrm{gr}$ : Sulsstanz gaben bei $769 \mathrm{~mm}$ und $19^{\circ}$.

$23 \mathrm{ccm} . \mathrm{N}=0,02676 \mathrm{gr}$. $=5,6 \% \mathrm{~N}$.

II a $0,5492 \mathrm{gr}$. Substanz gaben bei $762 \mathrm{~mm}$. und $19,5^{\circ}$

$19,3 \mathrm{ccm} . \mathrm{N} .=0,02126$ gr. $=4,04 \% \mathrm{~N}$.

II b $0,5247 \mathrm{gr}$. Substanz gaben bei $740 \mathrm{~mm}$. und $20^{\circ}$

$19,1 \mathrm{ccm} . \mathrm{N}=0,02126 \mathrm{gr} .=4,04 \% \mathrm{~N}$.

III a $0,5210 \mathrm{gr}$. Substanz gaben bei $728 \mathrm{~mm}$. und $20,5^{\circ}$

$20,0 \mathrm{ccm} . \mathrm{N}=0,02186$ gr. $=4,2 \% \mathrm{~N}$.

III b $0,5520 \mathrm{gr}$. Substanz gaben bei $741 \mathrm{~mm}$ : und $18^{\circ}$

19,2 ccm. $\mathrm{N}=0,021619 \mathrm{gr} .=3,92 \% \mathrm{~N}$.

VI $0,2452 \mathrm{gr}$. Substanz gaben bei $754 \mathrm{~mm}$. und $13^{\circ}$

22,2 ccm. $\mathrm{N}=0,02602 \mathrm{gr} .=10,6 \% \mathrm{~N}$.

Anmerkung. Nissl hatte gelegentlich eines Vortrages: Der gegenwärtige Stand der Nervenzellen-Anatomie und Pathologie (Centralbl. f. Nervenhlk. u. Psychiatrie) darauf aufmerksam gemacht, dass, wenn man Gehirne in Alkohol härtet, in den Alkohol etwas übertritt, was denselben gelb färbt, und ausserdem offenbar Cholesterin. Diese Substanzen finden sich nach einiger Zeit in einem sich am Boden der Gefässe absetzenden Schlamm. Die Untersuchung solcher Massen, die theils von Herrn Dr. Nissl, theils von Herrn Prof. Grashey dem Laboratorium freundlichst zur Verfügung gestellt waren, hat ergeben, dass neben Cholesterin noch $\mathrm{P}$ - und N-haltige Bestandtheile vorhanden waren, die beim Ausfallen des Cholesterins ebenfalls aus ihrer Lösung ausfielen und mit den oben beschriebenen Myelinsubstanzen identisch sein dürften. Auch Protagon enthielt jener Niederschlag: $\mathrm{C}=64,740_{j 0}, \mathrm{H}=11,25 \%$, $\mathrm{N}=2,7 \%, \mathrm{P}=0,721 \%$, offenbar durch die lange Dauer der Alkoholeinwirkung schon etwas zersetzt.

\section{Aetherextract des Eigelbs.}

Anhangsweise sei noch kurz die Lecithindarstellung aus Eigelb mittelst Acetonfällung des Aetherextractes mitgetheilt.

Das Eigelb von 50 Eiern wird in der Kälte mit Aether erschöpft. Die vereinigten Aetherfiltrate werden abdestillirt und vom Rückstand das darin enthaltene flüssige Oel bei Brutschranktemperatur abfiltrirt. Aufnehmen des möglichst ölfreien, eine zäh-schmierige, leicht schaumige, gelbe Masse bildenden Rückstandes mit wenig Aether und Fällen mit Aceton, solange als noch ein Niederschlag entsteht. Filtriren und Nachwaschen mit Aceton, bis das Waschaceton sich als cholesterinfrei erweist. Das Oel, sowie das gesammte im Eigelbextract enthaltene Cholesterin sind in Lösung geblieben und ins Filtrat übergegangen. 
Wiederum Aufnehmen des Niederschlages in möglichst wenig Aether oder Benzol und Hinzufügen der mehrfachen Menge absoluten Alkohols. Es fällt aus der anscheinend schon reinen Lecithinlösung durch Alkohol nach kurzem bis mehrstündigem Stehen ein weisser amorpher Niederschlag aus. Nach dem Abfiltriren des letzteren kann nunmehr das reine Lecithin entweder durch erneute Acetonfällung ${ }^{1}$ ) oder durch $\mathrm{Ab}$ destilliren des Alkohol-Aethers und Trocknen im Vacuum erhalten werden.

Der erwähnte, durch Alkohol bewirkte Niederschlag stellt nach seiner Reinigung (Umkrystallisiren aus heissem Alkohol, oder Lösen in Chloroform, Filtriren und Fällen mit Alkohol) ein krystallinisches weisses Pulver dar. Dasselbe ist unlöslich in Wasser, leicht löslich in Chloroform, schwerer in Aether und wenig löslich in Alkohol oder Aceton. Sein Sclımelzpunkt liegt bei $63^{\circ}$. Der Körper ist $\mathrm{N}$-frei und enthält keine anorganischen Bestandtheile. Seine Analyse ergab

$$
75,85 \% \text { C, } 12,44 \% \mathrm{H}, 11,71 \% 0 .
$$

0,1883 gr. Substanz gaben 0,2110 gr. $\mathrm{H}_{2} \mathrm{O}$ und 0,5237 gr. $\mathrm{CO}_{2}$.

Mit alkoholischer Kalilauge verseift, lässt sich, nach Ansäuern mit HCl, durch Aether eine sauere Substanz ausschütteln. Nach Verdunsten des Aethers bleiben alsdann schuppenartige weisse Massen vom Schmelzpunkt $62^{\circ}$ zurück, die dieselben Lösungsverhältnisse wie der ursprüngliche Körper zeigen. Letzterer ist demnach, wie leicht ersichtlich, Tripalmitin (berechnet $\mathrm{C}=75,93 \%, \mathrm{H}=12,40 \%$.)

Die Reinheit des auf oben beschriebene Weise dargestellten Lecithins wurde durch zahlreiche Phosphorbestimmungen sichergestellt, die stets einen P-Gehalt zwischen 3,7 und 4,1\% ergaben; eine Platinbestimmung ergab 11,1\% Pt.

1) Diese letzte Reinigung des schon nach den üblichen Methoden gereinigten Lecithins ist schon von Altmann empfohlen worden. 